\title{
Miroslav Krležas Mehrsprachigkeit
}

\author{
ISTVÁN FRIED \\ SZTE BTK Összehasonlító Irodalomtudományi Tanszék, H-6722 Szeged, Egyetem utca 2.
}

(Received: 5 December 2015; accepted: 11 February 2016)

\begin{abstract}
The aim of this paper is to demonstrate how M. Krleža takes advantage of the adaptabilities of multilingualism in his short story Hrvatski bog Mars. Soldiers from different linguistic areas served in the Austro-Hungarian multilingual army who needed to acquire the military language as well. However, establishing conversations with officers and soldiers of other languages was detained by misunderstandings and incomprehension, which lead to tragic and tragicomic consequences. The different social dialects created situations for talking at cross purposes, while the voided multilingualism of the upper classes signed cultural decadence, the coming of the end.
\end{abstract}

Keywords: Krleža, multilingualism, military discipline, linguistic behaviour of soldiers, intertextuality

Das im Jahre 1917 erschienene Buch Hrvatska rapsodija ist nicht nur ein emblematisches, die Gattung betreffend schwer einstufbares Werk des ostmitteleuropäischen Expressionismus. Es ragt auch durch die in der kroatischen Literatur ungewöhnliche Sprachlichkeit heraus und lässt die Kraft der Divergenz des seine letzten Tage erlebenden Reiches einerseits mit Hilfe der Absonderung der Sprachen und andererseits auch durch Zeichen eines einstmaligen Zusammengehörigkeitsgefühls spüren. Der scheinbar engagierte Narrator lässt die Personen in ihrer eigenen Sprache sprechen. In diesem Wirrwarr der Sprachen kommen folgende Gefühle vor: das Gefühl, seinen Weg zu verfehlen oder etwas nicht zu verstehen. Die im Kataklysmus ringenden Personen klagen umsonst über ihre Not und ihr Elend, jeder bleibt in seiner eigenen Einsamkeit. Es taucht kaum der Gedanke an eine Aussicht auf einen Dialog der Sprachen auf. Es scheint, als würden sich auch die Sprachen der einst zu einem Reich gehörenden Persönlichkeiten immer weiter voneinander entfernen.

Der erste Satz von Hrvatska rapsodija ist „Magyar állam vasutak“, der später als Abkürzung „M.A.V.“ auftaucht. Außer dem Schauplatz der Handlung wird auch ein Zeitpunkt erwähnt: „treća godina međunarodne vojne“ (KRLEŽA 1962: 385). Im kroatischen Text wird eine Aufschrift auf Deutsch zitiert, „k.u.k. Flugstation“ (KRLEŽA 1962: 389), und ein streitender Passagier kann die von ihm erwähnte Erscheinung nur so beschreiben, indem er den deutschen Ausdruck auch grammatisch in seine Muttersprache integriert: „Te njihove germanske ,Stilleben“- i ,Stubenglück'-dispozicije“" (KRLEŽA 1962: 394). Später wird das literarische Verhalten/ die literarische Äußerung, welches / welche nur mit Hilfe zweisprachiger Umschreibung definiert werden kann, in einem anderen Kontext wiederholt, was mit dem Stichwort „pseudocivilizacije“ zusammengefasst werden kann: „To je baš vaša 
laž, kao laž onih lirskih jadnika, koji pjevaju u ,Stillebenu“ i ,Stubenglücku'...“ (KRLEŽA 1962: 396). ${ }^{1}$ Das Wort „Stillleben“" wird im Weiteren interpretiert und gedeutet: mrtva priroda. Die literarische/künstlerische Lüge wird durch Einfügung des deutschen Sprachelementes verdeutlicht. Mit einem anderen Beispiel möchte ich betonen, dass der Text des Erzählers einerseits durch ungewöhnliche Äußerungen der anderen Personen ,zerfällt"“. Andererseits ,zerfällt" der Text auch durch die kroatisch sprechenden Personen und durch jene kulturellen, pseudo-kulturellen Erinnerungen, welche von anderen Regionen stammenden, oberflächlich adaptierten Wort- und Sprachelementen als charakteristisches Merkmal einer verzerrten Mehrsprachigkeit präsentiert werden.

In einer Kneipenszene der Rhapsodie (KRLEŽA 1962: 403) fordert ein aus Syrmien stammender Mann (Srijemac) den Zigeuner zum Geigenspiel auf. Der „primaš“ spielt zunächst einige Motive aus dem Pester Orfeum (,neki peštanski orfeumski motiv"), dann wird vom Mann aus Syrmien Folgendes zum Musizieren bestellt: „Rágyujtottam a pipára“. ${ }^{2} \mathrm{Zu}$ dieser Zeit verfasste Krleža zusammen mit den im Jahre 1921 publizierten Weltkriegsnovellen auch die Hrvatska rapsodija in einen Band (LASIĆ 1982) und ergänzte diese schließlich mit einem Wörterbuch, ${ }^{3}$ in dem die unverständlich gewordenen ungarischen, deutschen, lateinischen und französischen Ausdrücke mit Übersetzungen versehen wurden, und in dem auch einige kajkroatische Wendungen erklärt wurden. So hat eigentlich Krleža selbst die Aufmerksamkeit auf die sprachliche Mannigfaltigkeit des Bandes Hrvatski bog Mars gelenkt und erinnerte an die in Vergessenheit geratene vielsprachige Armee des vielsprachigen Reiches sowie an jene Schichtung, die bei der Konfrontation der Sprache von Personen aus unterschiedlichen Regionen, Gesellschaftsschichten, Positionen, mit verschiedenen Dialekten zum Vorschein kam sowohl für den zeitgenössischen Teilnehmer als auch für jene Leser, die die Geschehnisse des Ersten Weltkriegs bereits als Geschichte und Vergangenheit wahrnehmen.

Ich möchte ergänzen, dass Krleža während seines Lebens mit mehreren Sprachen vertraut war: Bekannt war ihm die kajkroatische Sprache, die er in seiner Kindheit von der Großmutter hörte, das Ungarische im ungarischen Bildungswesen (Fünfkirchen, Budapest), die deutsche Sprache der k. u. k. Monarchie sowie die die europäische Orientierung realisierende französische Sprache (LASIĆ 1982). Außerdem kann festgestellt werden, dass in Erzählungen des Ersten Weltkriegs, in denen das Soldatenleben zitiert wird, das Aufeinandertreffen der verschiedenen Sprachen ein durchaus effektives Mittel für die Beschreibung der Geschehnisse ist. Diese Szenen sind mal tragisch, mal tragikomisch oder eben auch satirisch.

\footnotetext{
1 „Bestritten und negiert wird die Ausdruckspotenz der modernistischen und sezessionistischen Literatur und Kunst" (KROLL 1990: 78).

${ }^{2}$ „In die Küche trat ich, meine / Pfeife wollt' mit Glut ich laben...“ (SCHWICKER 1889: 626, PETŐFI 1973); „Otvorenja je kuhinja / Na ognjistu vatratinja...“ (HoRVÁTH 1914: 8), prašime i zveketu paloša i konjskom topotu uz Zerkowitz-Petőfijev scherzo: „Befordultam a konyhába“ (KRLEŽA 1973: 197). Nicht B. Zerkovitz (1881-1948) komponierte die Musik, sondern man sang an die Melodie des Laura-Tschardasch von I. Frank (1825-?).

${ }^{3}$ „Tumač domobranskih i stranih riječi i pojmovima“ (KRLEŽA 1962: 419-466).
} 
Um zu dem obigen ungarischen Zitat zurückzukehren, erwähnt Krleža das „,petöfijanski scherzo“, die zweite Zeile eines Gedichts von Sándor Petőfi („Zapalio sam lulu...") als ungarisches volkstümliches Lied. ${ }^{4}$ Doch singt der Mann aus Syrmien mit Sicherheit nicht alleine, sondern mit anderen zusammen (,a i ostali s njima“), als würde das gemeinsame Gedächtnis einer gemeinsamen populären Kultur im gemeinsamen Elend zum Vorschein kommen. Nicht die ungarische Sprache ist hier das Hindernis der Verständigung, obwohl es natürlich durchaus möglich ist, dass die Melodie (die in der Tat mit der Volkstümlichkeit verbunden werden kann) die Singenden miteinander verbindet.

Der Titel einer Erzählung des Bandes ist vielversprechend: Magyar királyi honvéd novella (Kraljevska ugarska domobranska novela). Das Paradoxon des Titels ist, dass der Namensgeber eine „Institution“ ist, die in die ungarische und kroatische Geschichte eingetragen wurde, wenn auch mit gegensätzlichen Vorzeichen, insbesondere was die Geschehnisse vor und nach der Zäsur des Jahres 1868 betrifft. Ein satirischer Zielpunkt der Erzählung ist das, was der Erzähler als königlich ungarischen Offiziersjargon bezeichnet (KRLEŽA 1962: 48), ein künstlich verzerrtes Gebilde, das die leer gewordene, gedankenlose Kommunikation repräsentiert. In Wirklichkeit handelt es sich um eine „Nicht-Sprache“, die nicht bloß auf Gemeinplätzen baut, sondern das Abbild einer Lebensform ist, die wiederum auf jenen dummen (kretenska, idiotska), in der Armee eingebürgerten „Drill“" und übertriebene „Disziplin“ hinweist (KRLEŽA 1962: 56), durch deren Aneignung die Offiziere (oficiri) sozusagen die k. u. k. Monarchie aufrechterhalten konnten. Die Offiziere lernten Ausdrücke aus einem Wörterbuch (rječnik), die sie situationsgerecht anwenden konnten und so waren sie kommunikationsfähig, ohne eigene Beiträge, Meinungen zum Gespräch beizusteuern.

An einer anderen Stelle wird konkretisiert, was für Individuen die Armee führten (KRLEŽA 1962: 70). „Junkeri u atilama i dolomana marionete su iznakažene": atilla und dolmány sind ungarische Kleidungsstücke, marionett deutet nicht auf ihre Bewegung, sondern auf ihr Verhalten hin. Im weiteren Teil dieses Absatzes werden im Zusammenhang mit den besten Individuen dieser Kaste die Junker erwähnt, die an einem Pferderennen auf dem Lande (na kakvom našom provincijalnom steeplechaseu) gewonnen und eine Kassierfrau (kasafrajla) erobert haben (KRLEŽA 1962: 70). Die in den Text eingefügten Ausdrücke beleuchten jene Fremdheit und sprachliche Alterität, die sich im Grunde selbst offenbaren. Dies erfolgt mit Hilfe einer Diskrepanz, die vom Erzähler verursacht wird: Er verwendet vom kroatischen Text scharf abweichende Wörter, die von ihm als charakteristisch interpretiert werden und die bedeutende sachliche, gegenständliche und "geistige“ Faktoren zum Ausdruck bringen. Es wird jener Offiziersjargon (später wird kadetska škola erwähnt, vielleicht als Krležas persönliche Erinnerung) als exemplarisch dargestellt, der in die kroatische Redeweise eingeführt wird und als Sprache nur

${ }^{4}$ M. Engelsfeld sammelte die deutschen „Lehnwörter“, die in der Novelle Baraka Pet Be vorhanden sind: z. B. štimung, flaša, špital, rigcug, verter, ajnc, kajserjeger, vasistas usw. (ENGELSFELD 1964a: 95-98). 
in gewissen Kreisen eingebürgert wurde. Als adäquater Ausdruck, Jargon jenes frivolen, liederlichen (der Erzähler verwendet auch die Ausdrücke degeneriert und pervers-kränklich) Verhaltens, das wiederum auf das Verhalten zurückwirkt (ENGELSFELD 1964a: 95-98).

Zur Repräsentierung dieses Verhaltens hat jedoch die wohlbekannte, gängige Sprache keine Möglichkeit. Das in die Erzählung einkomponierte, zum Soziolekt gewordene „Kauderwelsch“ der Armee kann sogar grotesk wirken und als eine als Pamphlet wirkende Satire erscheinen. Der ungarische Titel der Erzählung führt uns in die Welt der Armee. Der Ausdruck honvéd muss aber erklärt werden. Krleža bringt den Ausdruck mit Landwehr in Zusammenhang und deutet darauf hin, dass domobran die Übersetzung für honvéd ist. Doch wird in den Erklärungen beim Stichwort domobranstvo (Landwehrwesen, honvédség) auf die Bedeutungswechsel hingewiesen, die sich zwischen 1848 und 1945 vollzogen. Die Armee der ungarischen Revolution von 1848 wird erwähnt, ${ }^{5}$ die die Marseillaise gesungen hat (in Wirklichkeit war dies nicht der Fall, sie haben es nämlich nicht gesungen) sowie das Gedicht Petőfis über einen ungarischen Jakobiner, die Carmagnole mit dem Titel Akasszátok föl a királyokat! [Hängt die Könige auf!] ${ }^{6}$ (KRLEŽA 1962: 424430) (auch dies wurde nicht gesungen). Krleža gibt in seiner Erklärung dem 1848er Gedicht Petőfis, das heftige Erregungen auslöste, einen kroatischen Titel, und deutet so darauf hin, dass er es von all dem drastisch trennt, was nach dem 1868er kroatisch-ungarischen Ausgleich geschah. Der Ausgleich (Nagodba) erhält einen selbstständigen Vermerk im Wörterbuch und die unglückseligen, ja tragischen Wendungen der kroatischen Geschichte nehmen mit 1918 kein Ende. Im Laufe der Auseinandersetzungen sticht ein unmarkiertes Zitat ins Auge: „nama treba jedan Mohač, jedan nova Udbina, jedan novi Solferino i Königgraetz“ (KRLEžA 1962: 439-440).

Krležas Mehrsprachigkeit kann auch als „Schicksalswenden“-Lektüre verstanden werden, deren literarische Erlebnisse er als Ausgangstext hernimmt und später in anderen eigenen Werken verarbeitet. Die oben erwähnte Zeile ist Endre Adys selbstplagende, die sich selbst einkapselnde Mentalität der Ungarn beschreibende, sich gegen jede Änderung wehrende Zeile aus dem Gedicht Nekünk Mohács kell [Wir brauchen Mohács]. ${ }^{7}$ Das Gedicht vergegenwärtigt die für die Ungarn ver-

5 „Unuci mrtvaca, što su pali na bečkom barikadama četrdesetosme, djeca Garibaldijevih stjegonoša, husiti, božji bojovnici, Jelačićovi graničari, madžarski Kossuthovi honvédi..." (KRLEŽA 1962: 285).

${ }^{6}$ Kraljeve vešajte, preveo B. Ćiplić im Jahre 1946, Die Könige hängt (PETÖFI 1938: 23-24). Im Leben des Dichters ist dieses Gedicht nicht erschienen (BABIĆ 1985: 502).

${ }^{7}$ Kroatisch in der Nachdichtung Krleža's:

Ako ima boga nek se ne smiluje na nas!

Mi smo na batine svikli i jučer i danas.

U nama se cigansko srce smije,

neka nas samo bije, bije, bije!

Ako ima boga, neka nas samo kara,

ja sam se rodio sa sudbinom Madžara! 
nichtend endende Schlacht gegen die Türken bei Mohács (1526), was zu den ungarischen Trauertagen gehört. Udbina ist ebenfalls ein Ort des Kampfes gegen die Türken, denn 1528 wurde es von den Türken belagert und danach folgten verheerende Verluste des Habsburgerreiches gegen die Italiener und Preußen (KRLEŽA 1961: 110-111).

Die Anwesenheit von Petőfi und Ady in Prosawerken und Essays des jungen Krležas ist wohlbekannt (vgl. VUJIČić 1959: 197-199, LASIĆ 1987: 336, 547-548, LŐKÖS 1982, LÖKÖs 1990, FRIED 2012). Doch diesmal wird der Titel von Adys Gedicht mit solcher Natürlichkeit herausgegriffen und zum Argument seiner eigenen Beweisführung gemacht, dass damit sowie mit der Tatsache, dass die Quelle der Herkunft des Gedichts nicht erwähnt wird, und gar nicht so erscheint, als sei es ein Zitat, es praktisch in Krležas Gedankengang integriert wird. Nur die Ortsnamen weisen auf eine eventuelle Übernahme hin. Anhand der historischen Ereignisse und Benennungen lassen die von Krleža konstruierten adeligen Namen ungarischer, österreichischer, italienischer Herkunft auf polyglotte Sprachlichkeit folgern, auf solche Transnationalität, bei der die Personennamen und die Sprache von $\mathrm{Za}$ gorje (VulETić 1975: 593-605) der Natürlichkeit der Volkssprache (in mehreren Erzählungen des Hrvatski bog Mars) der kajkroatischen Landschaft gegenübergesetzt wird: jener Mischsprachigkeit, die nicht die Folge der Polykulturalität ist, sondern aus dem oben genannten Jargon stammt. Die Offiziere und hohe Persönlichkeiten der österreichisch-ungarischen k.u.k. beziehungsweise der ungarischen Armee sind Repräsentanten einer „Anti-Kultur“. Diese Personen schöpfen aus dem Exerzierreglement und zu ihrer karikaturistischen Präsentierung verwendet der Erzähler das Hin- und Herflattern zwischen den Sprachen.

In der Erzählung Tri domobrana [Drei Honvéds] erklingen bei dem adeligen, eleganten Empfang Trinksprüche. Dem Karpatensieger gebührt ein dreimaliges „hura“ (auf Ungarisch: hurrá; Anfeuerung, Siegesruf), und dann geht die Diskussion darum, was im Pester Lloyd und in der Zeitung Neue Freie (Presse) gelesen werden kann. Ob die Revolution in Paris wohl ausbricht und wie das Orchester die „wunderbaren Akkorde“ der Operette Walzertraum von Oscar Straus (1907) spielt: „leise, ganz leise klingt's durch den Raum..." Aus den Ausführungen geht wahrscheinlich hervor, dass ähnlich zu H. Broch auch Krleža die Operetten der Monarchie als Produkte und Verursacher vom „Wertvakuum“ betrachtet (BROCH 1964: 59, 49) ${ }^{8}$ (wenn auch mit anderen Worten). Den berühmten Walzer der auch

\footnotetext{
Neka mi božji golub ne nosi maslinove grane, nek se obori na me, neka s munjom pane!

I ako ima boga, negdje od zemlja do neba, neka nas samo gazi, jer tako treba!
}

(KRLEŽA 1961: 111)

Vgl. noch ADY 1926: 51. Übersetzung von D. Kiš; Wir brauchen Mohács (ADY 1964: 7).

8 ,...so wurde die von Strauss begründete Operettenform ein spezifisches Vakuum-Produkt: als Vakuum-Dekoration hat sie sich nur allzu haltbar erwiesen, und ihr späterer Welterfolg kann geradezu als ein Menetekel für das Versinken der Gesamtwelt in das unaufhaltsam weiterwachsende Wert-Vakuum genommen werden“" (BROCH 1964: 59). 
heute gespielten Operette von O. Straus zitierend wird die Inhaltslosigkeit, Leerheit des Zusammenseins vom (Haupt)Adel veranschaulicht und zugleich jene Distanz, die zwischen den in den Krieg gezwungenen, unglückseligen Soldaten und den sorgenfrei und unbekümmert zechenden Herren besteht. Die oben erwähnten beiden Zeitungen erschienen in Budapest und Wien. Sie gehörten zu den halboffiziellen Presseorganen und gaben zugleich auch jener Monarchie-Kultur Raum, die Krleža eindeutig verneinte und zurückwies. Zu dieser Verneinung wird die in jener Epoche gefeierte Operette gegeben und Krleža erwähnt gerade die ersten zwei Zeilen des Refrains des Walzers, der zu den Wienerliedern gehörte. Der Text gibt mehrere Hinweise auf die von den Operetten popularisierte Lebensform beziehungsweise auf das popularisierte Lebensgefühl. Zur Veranschaulichung einige Zeilen aus der zweiten Strophe: „Nur fort aus den prunkenden Sälen, / Dorthin wo's uns besser gefällt, / Wo Wiener Walzer erzählen / von unserer heimischen Welt!“ (HeIn 2002: 52).

Der Kriegsschauplatz, das Soldatenschicksal und jene ungezwungene, leichtfertige Operettenwelt, die durch den Text und die Melodie des Walzertraums die heile Scheinwelt der Jahrhundertwende repräsentiert, könnten kaum besser dargestellt und veranschaulicht werden. Und dass im Text der Krleža-Novelle nicht eine etwaige kroatische Variante zu lesen ist, sondern F. Dörmanns Gedicht, ist nicht bloß ein Beitrag zur „Rhetorik“ der sprachlichen Grenzüberschreitung, sondern vielmehr die Widerlegung der These, dass die Vielsprachigkeit unbedingt und unreflektiert Werte darstellt. Hier wird gerade das Gegenteil dargestellt. Zugleich behält sich der Erzähler das „Recht“ vor, zwischen den Sprachen zu vermitteln und konstruiert die Möglichkeit des Aufeinandertreffens zwischen den verschiedenen Sprachen, wenn auch etwas einseitig. Dennoch kommt seine Perspektive, die von ihm gesteuerte Intertextualität, sein Dasein zwischen den Sprachen zur Geltung. Die Tatsache, dass der Erzähler mit mehreren Sprachen arbeitet, den Ort und Zeitpunkt, wo und wann eine Sprache zur Geltung kommt, selber bestimmt, untermauert und beweist, dass die Polykulturalität nicht seitens der teilnehmenden Personen, sondern seinetwegen und durch ihn im Text anwesend ist.

Es gibt Erinnerungen an Krleža, die als sein Hauptmerkmal erwähnen, mit welcher Sicherheit und Vertrautheit er sich zwischen den Sprachen bewegt, wie leicht er von einer Sprache auf eine andere umschaltet. M. Sperber schreibt: „Und dieser Krleža... der alles weiß, der mühelos von einer Sprache zu einer zweiten, einer dritten und vierten wechselt; der die Weltliteratur und die Weltgeschichte kennt, über alles auf dem laufenden ist" (SPERBER 1977: 36). Sperber erkennt die Ähnlichkeit Krležas mit J. Hašek und K. Kraus, ${ }^{9}$ und er hatte damit völlig Recht: Die Komposition der Erzähler-Sprache, die Vermischung der unterschiedlichen Soziolekte innerhalb eines Werkes, die Karikatur der Monarchie ist bei allen dreien die Darstellung der Armee, der Offizierskörperschaft. Die These ist treffend, wenn

9 „Karl Kraus, Jaroslav Hašek und Miroslav Krleža enthüllten das Gesicht der Weltkriegsmacher so, dass es einer von Gelächter und Todeskrampf geschüttelten Fratze glich.“ Vgl. noch: „Mit den unter dem Titel Kroatischer Gott Mars bald nach dem Krieg erschienenen Erzählungen errang Krleža eine einzigartige Position in der serbokroatischen Literatur" (SPERBER 1977: 34). 
auch etwas vereinfachend: Krleža beschrieb die Tragik des Krieges, Hašek die Komödie desselben (Lasıć 1987: 491). Wir können also akzeptieren, dass Švejk Anekdoten und Krleža längere Ausführungen verwendet, um die Geschichte „von unten" darzustellen. Bei den Betrachtungen der Freiwilligen von Zagorje ist diese Darstellung oft tragisch, insbesondere wenn es um ihre Jagd in den Tod geht. Parallel dazu wird das verzerrte Bild der mit bitterem Spott dargestellten Offizierskörperschaften oft satirisch oder ironisierend dargestellt.

In Krležas polyphoner Darstellung erhielt der Sprachwechsel eine äußerst wichtige Rolle. Die eingefügten Textteile (gerade aus dem Grunde, da sie überwiegend in einen längeren kroatischen Text eingeschoben werden) haben eine ungewöhnliche „Rechtschreibung“, das heißt das Buchstabenbild ist abweichend, sogar „unverständlich“, so dass die Lesbarkeit fragwürdig ist, und dabei hilft auch das am Ende vorzufindende Wörterbuch nicht. Einerseits aus dem Grunde nicht, weil nicht alle Fremdwörter, Ausdrücke, Namen, Verweise interpretiert werden, und andererseits weil das Hin- und Herblättern während des Lesens störend ist. Doch vielleicht ist gerade diese Ablenkung das Ziel des Erzählers: Er will herausfordern, dass sich der Leser nicht der Bequemlichkeit des leichten, sorglosen Blätterns, Lesens übergibt, sondern mit der ständigen Suche nach Erklärungen und Übersetzungen er sich ins Gelesene vertieft und sich somit auch den Text gestaltet. Der Krleža-Band ist nicht vollständig leserfreundlich: Er knüpft sehr eng an die präsentierte Zeitepoche an, und schöpft auch aus deren Kultur, sei es die ungarische und österreichische Literatur oder österreichische und ungarische populäre Kultur.

Zwischen 1917 und 1921 beherrschte noch ein Teil der Krleža-Leser die ungarische Sprache. Man kannte die von Krleža zitierten Gedichte, Texte und Melodien. Ein Großteil dieser Kenntnisse ging jedoch verloren, wie auch jene Sprache, die der Bestandteil der Mentalität der kroatischen Mittelschicht war. Die gemeinsamen Institutionen, das Zusammenleben sowie das ,agramerstvo“ - all dies ist größtenteils schon Vergangenheit. Daher benötigen die ins Kroatische übersetzten deutschen und ungarischen Ausdrücke Erklärungen. Der oben erwähnte Nachteil kann jedoch auch als Vorteil aufgefasst werden: Der heutige Leser stößt auf eine sprachliche Schicht, die ihn auf solch eine (egal, wie wir es bewerten: in eine dennoch) kulturelle Epoche aufmerksam macht, deren Mehrsprachigkeit wegen des Schematismus der Denkweise zwar „attackiert“ werden kann, weil sie ja in ein städtisches Kauderwelsch mündet. Auf der anderen Seite kann die Mehrsprachigkeit in der Zeit der Jahrhundertwende, in der Zeit der sich einschließenden Epigonenliteratur und der spätromantischen Leerheit, so erscheinen, als wäre sie zur großen Welt hin offen. Und wenn Krleža das provinzielle Denken kritisiert, nicht nur wegen des Sprachgebrauchs und der Makaronisprache, sondern wegen des durch die Sprache zum Ausdruck kommenden „falschen Bewusstseins“, dokumentiert Krležas Band (schon wegen der Notizen und Vermerke im Wörterbuch) die gegenseitige Berührung der Sprachen und den Austausch der Ausdrücke. Die groteske Szene einer Novelle stellt uns die Unverständlichkeit, die Tragikomik als Folge der unterschiedlich aufgefassten Sprachen vor Augen. Zuerst werden über ein Wort eines Gedichtes Witze gemacht, obwohl zunächst die deutsche, 
dann die ungarische und zuletzt die kroatische Variante vorgeführt wird („Hände waschen vor dem Essen, / Nach dem Stuhlgang nicht vergessen“; die ungarische Version ist nicht nur kürzer, sondern auch primitiver und der Rhythmus stockt: Egyél, igyál, de mindig elöbb mosdjál; kroatisch: „Peri ruke svagda prije jela, / peri poslije ispražnjenja tijela“). Daraufhin folgen die Tiroler: „Haha! Ist das aber wirklich dumm! Ist das dumm, dieses ,peri'! Was ist das, du, dieses - peri?" Woraufhin die kroatische Antwort: „Vasistas! Vasistas! Bedak zalupani. Niks! Niks! Daj mu ti reci, Štef, kaj pita! Ti si bil v Gracu! Kum! Kum! Šnaps!“ Man hört italienische, rumänische Wörter, dann klagt ein Kranker über seine Schmerzen. Die ungarische Reaktion: „Te! Mi az? Boli? Mindig ez a boli? Mi az a boli?“ (KRLEžA 1962: 282-284).

Unter den in Baraka Pet Be (Baracke 5B) zusammengeschlossenen armen Teufeln scheint die sprachliche Verständigung ein Hindernis zu sein, und in dieser verrückten Welt hilft nicht einmal die nonverbale Kommunikation. Über deren erfolgreiche Anwendung erfahren wir nämlich nichts. Die phonetisch fremd klingenden Wörter gehen verloren, und am Ende des Bandes machen die alphabetisch angeordneten Erklärungen das ruhige, entspannte Durchlesen des Buches auch nicht möglich. Dennoch ist dieses „Unverständnis“" etwas ganz anderes, als das „Unverständnis“ des Grafen Maksimilijan Axelrode, Komtur des Johanniterordens, Malteserordens, der die als Krankenhaus dienende Baracke errichtete. Wir folgen nicht seiner Geschichte, sondern der des todkranken Vidović (ENGELSFELD 1964: 87-100), denn mit dessen Tod geht diese die Oberschicht und die Unterschicht konfrontierende Darstellung zu Ende. Es kann nämlich kein Dialog zustande kommen. ${ }^{10}$ Die armseligen, kranken Personen verstehen einander nicht. Manche wollen Stille, manche Gespräche, so dass die Sprache auch hier trennt, da die Sprachen unterschiedlich sind, auch wenn die Schicksale ähnlich sind. Diese Sprachbarrieren verursachen Missverständnisse: Dasselbe wird anders ausgedrückt, die ungewöhnlich erscheinenden Lautgruppen (peri, boli, vasistas) rufen keine Assoziationen hervor. Andere Lautgruppen (Šnaps) können integriert werden, so dass sie Bestandteil der tagtäglich verwendeten Sprache werden. Gegensätze und Parallelitäten, Nichtverstehen und Bestrebungen sich zu verstehen können sowohl zur Zersplitterung, Zerrissenheit als auch zur Annäherung führen. Meistens kann in der Sprache des Erzählers der Ausgleich, die Annäherung erfolgen. In seiner Interpretation werden sogar die weit entfernten Momente zu Analogien.

Im Zuge einer Untersuchung erscheint ein Beweisstück (KRLEŽA 1962: 260), das in ein Tuch in den Farben der ungarischen Flagge eingehüllt war. Darauf die Aufschrift aus Mihály Vörösmartys berühmtem Gedicht (so wie wir es heute kennen), gefolgt von den ersten zwei Zeilen des Gedichtes in perfekter ungarischer Rechtschreibung Szózat: „Hazádnak rendületlenül / Légy híve, oh, magyar!“"11 In Klammern ist die kroatische Übersetzung beigefügt. Das nächste Beweisstück ist

10 „Trdak Vid govori i kajkavski i štokavski““ (ENGELSFELD 1964b: 109).

11 „Von Lieb und Treu zum Vaterland / bleib, Ungar, stets erfüllt..." (KulcSÁR SzABÓ 2013: 162). Vgl. noch: „učio napamet beskrajne heksametre o Moháču, o sigetskome Leonidi Nikoli Šubiću Zrinskome, o Vörösmartyjevom Arpadu s leopardovim krznom“ (KRLEŽA 1973: 184). 
eine Streichholzschachtel aus Istrien, darauf das Bild der beiden slawischen Apostel: Sankt Cyrill und Sankt Method. Die Bilder sind mit Wein übergossen. Vörösmartys Ode aus 1836 wurde zu feierlichen Ereignissen gesungen und ist ein hymnisches Gedicht. Cyrill und Method, wie auch der Text erwähnt, gehören zu den Heiligen aller slawischen Völker. Das Gedicht und die Heiligen sind in die Kategorie der Erhabenheit, Herrlichkeit einzuordnen, doch in dieser Szene von Tri domobrana werden sie zum Beweisstück degradiert und trivialisiert. Das Erhabene rutscht ins Untergeordnete, verliert somit in seiner materiellen Wirklichkeit die Heiligkeit. Der ungarische Text und die Konfrontation mit den slawischen Aposteln rief bei den Zeitgenossen Krležas wahrscheinlich ähnliche Assoziationen hervor, nämlich die von einer feierlichen Stimmung. Das ungarische und (diesmal) kroatische Erhabene wird materialisiert. Es entfernt sich von seiner wahren Funktion, und da es von Anfang an zum Gegenstand erniedrigt wurde, kann es sich nicht mehr vom Erniedrigungsmechanismus des Übergangs zu einem Gebrauchsgegenstand,,befreien“.

Der ungarische Text hebt die Bedeutung des Gedichts durch die Fremdheit der Sprache hervor, doch sei in Klammern angemerkt, dass als Erklärung die Rohübersetzung des ungarischen Gedichts beigefügt wurde. Ich muss jedoch hinzufügen, dass jede Rohübersetzung das dichterische Wesen wegnimmt. Das Wort wird mit einem Wort wiedergegeben und der Text somit seiner poetischen Funktion beraubt; das Gedicht und seine Rohübersetzung sind von vornherein Gegensatz, Entfremdung. Um daran zu erinnern, wo Vörösmartys beide Gedichtzeilen und das Bild der slawischen Apostel im Text auftauchen, erinnern wir an die Ausrüstung des honvéd (domobran). Dessen Ausrüstung wurde eigentlich unnötig geschildert, da sie für ihn völlig uninteressant zu sein scheint, geschweige denn er eine ,nationale“ Sakralität damit verknüpft.

Es muss auch betont werden, dass der Besitzer ein völlig gleichgültiges und sprachlich ,unwichtiges“ Verhältnis zu diesen Gegenständen hat. (Das ungarische und slawische „Symbol“" sagen ihm auch nichts.) Das zeigt, dass er nichts mit den offiziellen Sprachen zu tun hat, egal, ob diese in Text- oder Bildform erscheinen. Ich zitiere vom letzten Teil eines längeren Absatzes. Der Erzähler lässt uns nach einem (deutsch-kroatischen) mehrsprachigen Dialog in einer erlebten Rede nachdenken. Er lebt sich in die Gedanken einer Person hinein (KRLEŽA 1962: 92), über die Vorteile des Großstadtlebens, die schöne Seite des Aufenthaltes in einem Krankenhaus. Gewöhnlich formulieren Krležas Erzähler ihre Gedanken in langen Sätzen, doch hier sind die kurzen Sätze, das dichte Hintereinander der Gedankenassoziationen charakteristisch. Im Sinne der zitierten Schauplätze ragen die früher nur kurz erwähnten fremdsprachigen Erinnerungen an dieser Stelle durch ihre Mischsprachigkeit hervor: „Pa nekamo u koju lijepu bolnicu! U sanatorij u Beč! Ili Peštu! Lijepa bolničarke, fine gospođe! Opet kakva gospođa Loebl! Ili kakva jaj de feš Peštanka. Gerbeaud. ${ }^{12}$ Opera. Hotel Fijume! Little lady!““

\footnotetext{
${ }^{12}$ Gerbeaude: Konditorei im fünften Bezirk, gegründet im Jahre 1858, Eigentümer vom Jahre 1884: E. Gerbeaude (vgl. noch KRLEŽA 1973: 195).
} 
Der Leser findet nicht jedes Wort in den Erklärungen des Wörterbuchs, insbesondere sind keine auf konkrete Orte, Plätze hinweisenden Benennungen aufzufinden. Doch sowohl der ganze Absatz als auch die wenigen zitierten Zeilen wiesen auf den Ausgleich der Bedeutungswidersprüche in der Lebensauffassung hin, mit Hilfe der getreulich darstellenden Mehrsprachigkeit. Das deutsche Wort im ungarischen Ausdruck mit kroatischer Rechtschreibung: feš (fesch) ist schon an sich vielsagend. Dazu kommen noch Pester Schauplätze, wie Gerbeaude mit französischer Herkunft, das bis zum heutigen Tag eine der eleganten Konditoreien der Pester Innenstadt ist, ein Treffpunkt der „,vornehmen“ Gesellschaft. Auch das (Budapester) Opernhaus wird zu einem Ort, wo man repräsentieren und Logen besuchen kann, sowie das Hotel Fijume (in ungarischer Rechtschreibung: Fiume). ${ }^{13}$ An letzter Stelle der Aufzählung steht Little lady, und somit wird (falls der Leser die Entdeckung noch nicht gemacht hätte) auf die leichtere Unterhaltung hingewiesen. ${ }^{14}$ In der Träumerei erscheinen die schönen Krankenhäuser, die schönen Krankenschwestern, das Wiener Sanatorium, und die Einzelheiten der Träumerei werden durch verschiedene sprachliche Fragmente in Form gesetzt, wie auch das aus dem kroatischen Text herausragende Element, dessen Paradoxon mit Hilfe der Gegenüberstellung der Honvéds von Zagorje hervortritt. Was jenseits der Träumereien liegt, schreibt Vježbovnik za kr. ugarsko domobransko pješactvo vor, ${ }^{15}$ und auf ihre Budapester Ausgabe von 1912 nehmen die Erklärungen Bezug, die mit großer Wahrscheinlichkeit auch von Krleža benutzt wurde.

Der relativ lange, über Ironie verfügende Titel des Buchs summiert jenen Verhaltenskodex, wonach der domobran zu dienen hat. Eine der wundervollsten kroatischen Bücher, ein fantastisches Buch, dessen sprachliche Charakteristika - „lingvistička komedija“" oder auch ,paranoidni ričet" - jenes System darlegt, an dem sich die Armee auszurichten hat. Und obwohl der Titel nicht mit der Möglichkeit der Mehrsprachigkeit spielt, wird durch das Kauderwelsch der Armee mit Hilfe der sich hinter dem Buch spannenden Faktoren der Welt- und Landanschauung angedeutet, dass Vježbovnik das sprachliche Werk der am Ausgleich teilnehmenden Unionisten ist. Die aus dem Buch herausgegriffenen Zitate dienen als Beweismaterial zur Charakterisierung der eine abstrahierte Ordnung dokumentierenden Reglements, deren Absurditäten sowohl sprachlich als auch die Charakterisierung betreffend in den Novellen von Hrvatski bog Mars wiederkehren. Teils von hier, teils aus dem den Wortschatz der Armee zusammenfassenden Wörterbuch stammen die ins Kroatische eingebürgerten deutschen Ausdrücke bzw. Abkürzungen: A. D. - Außer Dienst; angrif - Angriff, abtajlunk - Abteilung, A.O.K. - ArmeeOberkommando, was einige als Alle ohne Köpfe verdrehten, befel - Befehl, cimerZimmer, cugsfirer - Zugsführer, drot - Draht. (Drot könnte auch vom ungarischen

${ }^{13}$ Hotel Fiume und Kaffeehaus in Ofen (Buda), im zweiten Bezirk, Lánchíd utca (Kettenbrückengasse) 12.

${ }^{14}$ Ein populäres Couplet um 1910. Vgl. noch: ,pjevao najnovji Medgyaszay-kuplet: ,Little lady, o yes, o yes‘. Vilma Medgyaszay (1885-1972): Chansonette“ (KRLEŽA 1973: 197).

${ }_{15}$ Vježbovnik za kr.ug. domobransko pješactvo. Treća izdanja. Budapest, 1875. Weitere Ausgaben: 1895, 1900, 1901, 1912, 1916. 
drót abgeleitet sein. Die erste ungarische Aufzeichnung kann auf das 17. Jahrhundert zurückdatiert werden und gehört zu den bayrisch-österreichischen Lehnwörtern.)

Solch eine Liste können wir auch aus den ungarischen Wörtern und Ausdrücken zusammenstellen. (Am Rande vermerkt: Einige französische, italienische und lateinische Wörter müssten auch erklärt werden.) Die Reihe der ungarischen Ausdrücke wird von Fluchwörtern, Schimpfereien eröffnet: Az apád istennét! (korrekt: istenit); Csönd! - madžarska psovka: Boga ti očina! Kuš!; baka - prost vojnik, redov; betežan - bolestan u kajkavskom, od madžarskog: beteg (krank); čaka, čako [...] od madžarskog: csákó [...] Lagermütze; doboš - bubanj, dobošar, dobošarevica. Madjarizmi: ember - madžarski: čovjek; dazu kommen noch: die Aufschrift der M.A.V. (Ung. Staatsbahn); 36 ember (Mann), 6 ló (Pferde); melin, kajkavski mlin. Mađarizam: malom (Mühle); nemeš (nemes), madžarski: plemič (Nobel); Te! Mi az?: Ti! Što to znači?

Die Liste der Erklärungen ist keinesfalls vollständig, doch so viel ist sicherlich ersichtlich: Einerseits werden die in der damaligen Allgemeinsprache (der Armee) öfter vorkommenden Wörter, andererseits die in der kroatischen (oder in der kajkroatischen) Sprache eingebürgerten Wörter im Wortverzeichnis des Anhangs erklärt. Im ersten Fall muss erwähnt werden, dass die Ausdrücke über einen solchen kulturgeschichtlichen Hintergrund verfügen, zu dessen Verständnis der heutige Leser durchaus weitere Erklärungen braucht. Mit deren Hilfe wird der Umriss, das Armeebild und im Allgemeinen die Kultur der Jahrzehnte nach dem Ausgleich erläutert und nahegelegt. Dem Leser wird somit jene Gegenkultur präsentiert, gegen die dieser Band und die im selben Jahrzehnt publizierte ganze Reihe der Krleža-Essays ihren eindeutigen Standpunkt erklären. Im Fall von Gott erhalte hält Krleža seinen heftigen Zorn zurück und offenbart, dass die Musik von Haydn komponiert wurde und dass es eine Variante von God save the King ist (KRLEŽA 1962: 432).

Noch heftiger ist die Deutungsstrategie der Erklärung der Gassenhauer-civilizacija (KRLEŽA 1962: 431-432). Ein Gassenhauer ist ein Lied, das in allen Gassen gesungen wurde. Ende des 19. Jahrhunderts war Gassenhauer trivialisiert und erhielt somit gegenüber dem Wienerlied eine Gattungskategorie von (lyrisch-musikalisch) geringerem Niveau. Krležas Erklärung setzt Gassenhauer mit der banalen Operettenarie gleich, so ist die Gassenhauer-Zivilisation identisch mit der Wiener Operetten-Zivilisation, der Franz-Josefschen Bordellen-Zivilisation, dem Prater, der Operette, dem Walzer, dem Zirkus. In diesem Sinne ist der Ausgleich in der Österreichisch-Ungarischen Monarchie bestimmend, der von Lügen und Falschheit geprägt wird.

In den Jahren zwischen 1917 und 1921 lebte noch die Ansicht der letzten Jahre, in der Krleža (ich wiederhole mich an dieser Stelle) seine Ansichten mit Hašek und Kraus teilte, die „kulturelle“ Erklärung für den Zerfall der Monarchie suchte und die Gründe sowie Symptome für die generell gewordene Wertlosigkeit im Band Hrvatski bog Mars aufzeigte. Krleža war dort der Ansicht, dass (wie im Titel des Bandes) die Geschichte der Paradoxa der Kriegsjahre genauso als Ur- 
sache angeführt werden muss, wie die Sprachkrise, für die er als Beispiel die verzerrte Form der Mehrsprachigkeit heranzog. Er veranschaulichte die Entfremdung der zusammenlebenden Nationen mit Hilfe der sprachlichen Entfremdung, während er auch nicht vergessen hatte, dass nicht bloß die Erfahrung einer als oberflächlich gedachten Sprachberührung formuliert wird, sondern auch die einer im Tiefen vorhandenen sprachlichen Beziehungsgeschichte. Als Beweis können wir Erklärungen über die ungarischen Lehnwörter aus der kroatischen/kajkroatischen Sprache lesen. Mit den Wörtern, Ausdrücken, Fluchwörtern und ironischen Kommentaren wird auch jene kulturelle/pseudokulturelle Scheinwelt klar, die gerade mit den karikaturhaften Stimmen der Mehrsprachigkeit in erster Linie in die Sprachweise der Personen in der Erzählung eingeschoben wird. Der Erzähler distanziert sich von seiner eigenen Weltanschauung und seiner Sprache. Womit er sich jedoch identifizieren kann, ist die Schicksalsgeschichte seiner in die Armee gezwungenen Honvéds aus Zagorje. Der Erzähler ist der Ansicht, dass die kajkroatische Sprache geeignet ist, dies zum Ausdruck zu bringen, und diese Sprache kann nicht nur aus dem Munde der einzelnen Personen in der Erzählung, sondern auch vom Erzähler selbst gelesen werden.

Eine Person in der Erzählung Bitka kod Bistrice Lesne [Die Schlacht bei Bistrica Lesna] spricht sowohl die štokroatische als auch die kajkroatische Sprache. Mit dem Dialekt beglaubigt er einerseits seine Zugehörigkeit (VuLETIĆ 1975) und andererseits seine Existenz zwischen den Sprachen. In derselben Erzählung werden als Quelle des Wortbestandes eines Briefes zwei Dichter der kroatischen Epigonenliteratur des 19. Jahrhunderts angegeben, Harambašić und Badalić, ${ }^{16}$ um anzudeuten, dass die populären Dichter der zweiten Hälfte des 19. Jahrhunderts auch nicht imstande waren, sich selbst von der Gefahr des Sprachverlustes zu distanzieren. Der Erzähler hebt die Illusionen der einmarschierenden Soldaten mit einem eingeschmuggelten Literaturhinweis in die Welt der Träumerei. Was den entlehnten Begriff betrifft, wird auf die Jahrhundertwende-Thematik von Schein und Sein hingewiesen: ,,u prve gradske dane takvom Šlarafijom“, die in der Stadt verbrachten ersten Tage erscheinen den in die Armee einrückenden jungen Bauern als Schlaraffenland. An dieser Stelle kann vielleicht H. Manns Roman Im Schlaraffenland (1900) beziehungsweise das Vorkommen des Schlaraffenland-Motivs, angefangen mit S. Brant und H. Sachs bis hin zu den Grimm-Märchen, erwähnt werden. Der Erzähler sucht für die in den ersten Tagen des Krieges noch verblüfften Jünglinge in einem ziemlich breiten kulturellen Kontext Platz, und mit der Einfügung von Šlarafija weist er sprachlich auf die Naivität und auf die nicht ganz natürliche Art dieser Verwunderung hin.

Die Mehrsprachigkeit in Krležas Band Hrvatski bog Mars lenkte auch die Aufmerksamkeit der sprachwissenschaftlichen (HADROVICs 1985) und stilistischen Forschung (ENGELSFELD 1964b) auf sich. Die Forschung wies darauf hin, über wel-

${ }^{16}$ A. Harambašić (1861-1911), Dichter, Rechtsanwalt, Politiker; H. Badalić (1851-1900), Librettist der Oper Nikola Šubić Zrinski von Ivan Zajc (1852-1914); ,,iz ljubavnog listara neke harambašićove-badalićevski izraze..." 
che Bedeutung ein mit Hilfe der Rechtschreibung eingebürgertes oder gerade mit der ursprünglichen Rechtschreibung veröffentlichtes deutsches oder ungarisches Wort, ein Ausdruck, Name, geschichtliche oder kulturgeschichtliche Hinweise im kroatischen Text verfügen können. Aus den von mir erwähnten Beispielen geht wahrscheinlich hervor, dass bei der Vorstellung dieses Werks (oder anderer Werke) von Krleža wir eher von den negativen Bezügen der Mehrsprachigkeit und Polykulturalität sprechen sollten, als über die verschiedenartig geschichtete Multikulturalität einer mehrsprachigen Gesellschaft oder eines mehrsprachigen Staates (NYOMÁRKAY 2012).

Wie der Text gestaltet wird, hängt auch davon ab, wie der Erzähler oder seine Figuren fremdsprachige Elemente aus der Gemeinsprache oder den Soziolekten in den Text einfügen. Wörter können - wie im Fall der Lehnwörter - dauerhaft in eine Sprache aufgenommen werden und zu deren Bereicherung führen. Zahlreiche Elemente der übernommenen, im Wesentlichen aus der Amtssprache stammenden Wörter gehören zu verschiedenen gesellschaftlichen Schichten, daher können sie jedoch leicht in Vergessenheit geraten. Die an die deutsche (österreichische) und ungarische Literatur und Kultur anknüpfenden Texte benötigen ebenfalls eine eingehendere, tiefere Analyse: Die Komplexität von Krležas Intellektualität und Bildung ist die Konsequenz der dichten Intertextualität dieses Bandes und anderer Werke des Verfassers.

\section{Literatur}

ADY 1926 = ADY Andreas: Auf dem Flammenwagen der Lieder. Übertr. Albert HetényiHeidelberg. Budapest, 1926.

ADY 1964 = ADY Endre: Pjesme. Izbor i prevod: Danilo Kiš. Beograd, 1964.

BABIĆ 1985 = BABIĆ Sava: Kako smo prevodili Petefija. Novi Sad, 1985.

BROCH 1964 = BRoch Hermann: Hofmannsthal und seine Zeit. München, 1964.

Engelsfeld 1964a = Engelsfeld Mladen: Miroslav Krleža „Baraka Pet Be“ (Primjedve uz stil). In: FrAngeš Ivo, FlaKer Aleksandar (ured.): Krležin zbornik. Zagreb, 1964. 87-100.

Engelsfeld 1964b = Engelsfeld Mladen: Još o stilu Miroslava Krleže. In: Frangeš Ivo, FLAKER Aleksandar (ured.): Krležin zbornik. Zagreb, 1964. 101-114.

FRIED 2012 = FRIED István: Überlegungen zu M. Krležas Bezügen zur ungarischen Literatur. Studia Slavica Hung. 57 (2012): 429-439.

HADROVICS 1985 = HADROVICS László: Ungarische Elemente im Serbokroatischen. Budapest, 1985.

HeIN 2002 = HeIN Jürgen (Hg.): Wienerlieder. Von Raimund bis Georg Kreisler. Stuttgart, 2002.

KRLEŽA 1961 = KRLEŽA Miroslav: Eseji 1. Knj. 1. Zagreb, 1961.

KRLEŽA 1962 = KRLEŽA Miroslav: Hrvatski bog Mars. Zagreb, 1962.

KrLeŽA 1973 = KrLEŽA Miroslav: Glembajevi. Proza. Sarajevo, 1973.

Kroll 1990 = KROLL Walter: Aspekte der Mythos-Rezeption am Beispiel „Hrvatska rapsodija“. In: LAUER Reinhard (Hg.): Künstlerische Dialektik und Identitätssuche. Literaturwissenschaftliche Studien zu Miroslav Krleža. Wiesbaden, 1990. 249-267. 
KulCSÁR SzABÓ 2013 = KulCSÁR SzABÓ Ernő (Hg.): Geschichte der ungarischen Literatur. Eine historisch-poetische Darstellung. Berlin, 2013.

LASIĆ 1982 = LASIĆ Stanko: Krleža. Kronologija života i rada. Zagreb, 1982.

LASIĆ 1987 = LASIĆ Stanko: Mladi Krleža i njegovi kritičari (1914-1924). Zagreb, 1987.

LÖKÖS 1982 = LÖKÖs István: Miroslav Krleža szépírói élményvilágának magyar rétegeiröl. In: SzeLi István (szerk.): Délszláv-magyar irodalmi kapcsolatok 1. Újvidék, 1982. $252-258$.

LÖKÖs 1990 = LöKÖs István: Die Frage der Ady-Rezeption in Krležas Werk. In: LAUER Reinhard (Hg.): Künstlerische Dialektik und Identitätssuche. Literaturwissenschaftliche Studien zu Miroslav Krleža. Wiesbaden, 1990. 249-267.

NYOMÁRKAY 2012 = NYOMÁRKAY István: Die geistige Verwandtschaft der ostmitteleuropäischen Sprachen. Studia Slavica Hung. 57 (2012): 25-35.

PETÖFI 1908 = Petőfijeve pjesme. Preveo Béla Horváth. Subotica, 1908.

PETÖFI 1938 = PETŐFI Alexander: Lyrische und epische Dichtungen. Übers. Ludwig Landgraf. Budapest, 1938.

PetŐFi 1973 = Petefi Sándor: Pesme. Izbor: Mladen Leskovac. Beograd, 1973.

SCHWICKER 1889 = SCHWICKER Johann Heinrich: Geschichte der ungarischen Literatur. Leipzig, 1889.

SPERBER 1977 = SPERBER Manès: Bis man mir Scherben auf die Augen legt. All das Vergangene. Wien, 1977.

VujIČıć 1959 = Vujicsics D. Sztoján: Ady et les écrivains serbo-croates. Acta Litteraria Hung. 2 (1959): 185-201.

VulETIĆ 1975 = VulETIĆ Branko: Kajkavski elementi u krležinim štokavskim tekstovima. In: Krolo Ivan, MatKović Marjan (ured.): Miroslav Krleža 1973. Zagreb, 1975. 595605. 